Vol. 5, n' 1 | 2001

Varia

\title{
Esquisse d'une économie de l'illicite. Le marché parallèle de la viande à Lyon pendant le Carême (1658-1714)
}

Lauréate du Prix Herman Diederiks 2000 / The Herman Diederiks Prize Essay for 2000

\section{Anne Montenach}

\section{(2) OpenEdition}

\section{Journals}

Édition électronique

URL : https://journals.openedition.org/chs/775

DOI : $10.4000 /$ chs. 775

ISSN : 1663-4837

\section{Éditeur}

Librairie Droz

Édition imprimée

Date de publication : 1 janvier 2001

Pagination : 7-25

ISBN : 2-600-00607-9

ISSN : 1422-0857

\section{Référence électronique}

Anne Montenach, « Esquisse d'une économie de l'illicite. Le marché parallèle de la viande à Lyon

pendant le Carême (1658-1714) », Crime, Histoire \& Sociétés / Crime, History \& Societies [En ligne], Vol. 5, $\mathrm{n}^{\circ} 1$ | 2001, mis en ligne le 06 avril 2009, consulté le 24 mars 2022. URL : http:// journals.openedition.org/chs/775; DOI : https://doi.org/10.4000/chs.775 


\title{
Lauréate du Prix Herman Diederiks 2000 The Herman Diederiks Prize Essay for 2000
}

\author{
Esquisse d'une économie de l'illicite \\ Le marché parallèle de la viande à Lyon pendant le Carême \\ (1658-1714)
}

\section{Anne Montenach ${ }^{1}$}

Cette étude analyse, par le biais de sources judiciaires, les liens entre normes et pratiques dans le cadre précis du marché parallèle de la viande en Carême à Lyon dans la seconde moitié du XVII ime siècle. Elle présente le système officiel de la ferme avant d'esquisser l'inscription spatiale et sociale de ce type d'économie souterraine que représente le marché noir de la viande. L'étude des confrontations physiques et verbales entre les acteurs de ce commerce et la justice est par ailleurs l'occasion de mesurer le rôle des défiances et des solidarités communautaires et d'analyser les stratégies de légitimation mises en ouvre par les fraudeurs. Elle montre enfin comment les pratiques peuvent être à l'origine d'une réécriture factuelle ou formelle de la loi.

The purpose of this paper is to analyze, using legal sources, the relationship between norms and practices within the specific framework of the unofficial meat market during Lent in Lyon in the second half of the $17^{\text {th }}$ century. It first presents the official system of the meat trade, then proceeds to discuss the geographical and social aspects of the black market. The examination of the physical and verbal confrontations between the participants of this trade and the authorities further provides an opportunity to measure the role of mistrust and community solidarity and to analyze the legitimizing strategies used by the lawbreakers. Finally, this paper demonstrates how practices can lead to a factual or formal rewriting of the law.

L es réflexions menées sur les interactions entre les individus et la norme ont montré que les contraintes formelles pouvaient constituer des obstacles à l'action des hommes. Elles ont aussi mis en valeur, à l'inverse, le fait que les pratiques interfèrent en permanence avec la norme: en les contournant, voire en les

1 Doctorante en histoire moderne à l'Institut Universitaire Européen de Florence (a bénéficié d'une bourse du ministère des Affaires étrangères dans le cadre du programme Lavoisier); actuellement attachée temporaire d'enseignement et de recherche (ATER) à l'Université de Provence (AixMarseille I). Sa thèse en cours, porte sur l'approvisionnement et les consommations alimentaires à Lyon au XVII ${ }^{\text {ème }}$ siècle (sous la direction du professeur Laurence Fontaine). Elle a publié: «La boutique au cour du commerce alimentaire à Lyon au XVII ${ }^{\text {ème }}$ siècle: entre économie légale et marchés parallèles ", in Natacha Coquery (dir.), La boutique et la ville. Commerces, commerçants, espaces et clientèles $X V I^{\text {ème }}-X X^{\text {ime }}$ siècles, Actes du colloque des 2,3 et 4 décembre 1999, Tours, Publications de l'Université François Rabelais, 2000, 505 p.,pp. 31-44; « Du marché idéal aux marchés réels: les formes du contrôle et de l'échange dans le cadre des marchés alimentaires lyonnais au XVII ${ }^{\mathrm{e} m o}$ siècle », in Foires et marchés dans l'intégration des économies européennes XIII ${ }^{2 m e}-X V I I{ }^{2 e m e}$ siècles, Actes de la XXXII ${ }^{\mathrm{a}}$ Settimana di Studi, Prato, Istituto Internazionale di Storia Economica F. Datini, 8-12 mai 2000 (à paraître). 
exploitant, les individus finissent, en fait sinon en droit, par transformer les normes qui régissent la vie en société2. L'étude de la boucherie de Carême sous l'Ancien Régime permet ainsi d'analyser les différents processus qui font qu'une règle pourtant simple et claire en apparence est attaquée de tous côtés et finalement vidée de son sens ${ }^{3}$. Depuis le haut Moyen-Âge, les prescriptions de l'Église catholique interdisent en effet la consommation de viande pendant le Carême 4 . Notons à ce propos que même si le blé continue d'occuper (au moins jusqu'à la décennie 1740-1750) une place essentielle dans l'alimentation des populations de l'Europe moderne, la viande constitue alors, par ses apports protéiques, une part non négligeable du bol alimentaire ${ }^{5}$. Pendant cette période de quarante jours précédant Pâques, les boucheries de Lyon sont fermées et le monopole de la vente de la chair destinée aux personnes dispensées de jeûne est confié à un seul boucher. À Paris, c'est l'Hôtel-Dieu qui détient ce privilège et $R$. Abad a pu étudier, à partir du nombre d'animaux qui y étaient abattus pendant cette période, l'évolution de la consommation de viande en Carême comme un nouvel indicateur du recul de l'observance religieuse des Parisiens au XVIII ${ }^{\text {ème }}$ siècle ${ }^{6}$. Or il est aussi intéressant d'aborder la question de la consommation illicite de viande en Carême autrement que sous l'angle d'une transgression des interdits religieux. À Lyon, pendant ces quarante jours, un véritable marché parallèle de la viande fonctionne en marge du système d'adjudication officielle. Au travers des sources produites dans la seconde moitié du XVII ${ }^{\text {ime }}$ siècle par la surveillance policière et judiciaire - qui par ses enjeux renvoie effectivement aussi au rapport à la religion - il est utile de s'interroger sur l'économie de ce marché parallèle dans ce qu'elle nous dit des relations entre les individus et la norme. Procès-verbaux et interrogatoires permettent ainsi d'appréhender l'inscription spatiale de ce marché noir et d'esquisser au sein de l'espace urbain et périurbain une géographie de l'illicite. Ils mettent aussi en lumière les motivations des contrevenants à la loi - agents de ce commerce ou simples consommateurs - et les multiples stratégies que ceux-ci mettent en œuvre pour utiliser les failles du système de surveillance. Les gestes, les discours, mais aussi les modalités concrètes d'application de la loi éclairent enfin, au travers de cet exemple concret du Carême, le champ des négociations possibles entre les individus et la règle.

\section{LES INTERDITS RELIGIEUX ET LE SYSTÈME DE LA FERME}

Depuis le IX ${ }^{\text {ème }}$ siècle, l'Église prescrit le jeûne - et en particulier l'interdiction de consommer de la viande, du gibier, de la volaille ou des cufs - pendant les quarante jours qui séparent le mercredi des Cendres de Pâques. Le Carême est en effet conçu par l'Église comme un temps de pénitence et le jeûne considéré comme « une œuvre des plus essentielles de cette pénitence». En effet, «c'est par le jeûne que les

2 Revel (1995), Cerutti (1995).

3 La question du commerce illicite de la viande en Carême n'a pour l'instant pas fait l'objet d'études. Sur d'autres aspects de la fraude et de la délinquance à petite échelle, voir Garnot (1998).

4 Péronnet (1994).

5 Flandrin, Montanari (1996).

6 Abad (1999). 
justes sont conservez dans l'heureux état de la grâce, que les foibles sont soutenus contre les tentations de la chair, du monde et du démon, et que les pécheurs retournent à Dieu par la mortification de leur corps et le changement de leur cœur». C'est pourquoi l'Eglise, suivant en cela l'exemple donné par le Christ, «en a déterminé le temps aux jours qui precedent la solemnité de sa résurrection, afin que nous puissions célébrer avec un corps mortifié et un esprit purifié les divins mystères de nôtre rédemption $»^{7}$. L'interdiction de consommer de la viande ne peut de ce fait être annulée qu'en cas de nécessité absolue. C'est par exemple le cas lorsque la peste frappe Lyon en 1629: l'archevêque octroie alors la permission, non seulement aux malades, mais à tous ceux «qui se trouveront avoir besoin d'en manger», de se fournir de chair ${ }^{8}$. De la même façon, la crise de l'hiver 1709 incite les autorités religieuses à permettre aux fídèles du diocèse de manger de la viande «tous les dimanches, lundis, mardis et jeudis de chaque semaine du Caresme prochain, à commencer au premier jeudi qui suit immédiatement le jour des Cendres jusques au dimanche des Rameaux exclusivement $»$.

En dehors de ces années d'exception, les quatre boucheries de la ville sont fermées pendant toute la durée du Carême ${ }^{10}$ et le monopole de la vente de la viande, destinée à ceux qui ne peuvent faire maigre (enfants, vieillards, malades, femmes en couche, etc.), est octroyé aux enchères à l'un des bouchers de la ville par les recteurs de l'hôpital de la Charité (ou Aumône générale). Tous les ans à la fin du mois de janvier ou au début de février, les recteurs de l'Aumône générale envoient au Consulat une députation, pour le prier de mander un de ses représentants pour «assister à la délivrance de la ferme de la permission de vendre de la chair durant le caresme prochain en la forme et manière accoustumée $»^{11}$. Le bureau de l'Aumône générale se réunit alors en présence des recteurs de l'Hôtel-Dieu, du lieutenant général ou de l'un des magistrats de la sénéchaussée, du procureur du roi et d'un représentant de l'archevêque ${ }^{12}$. Comparaît aussi «la plus grande et sayne partie des bouchers de la ville», convoquée par affiches et par le crieur juré de la ville (M. Garden évoque - pour le XVIII ${ }^{\mathrm{e} m e}$ siècle - l'obligation de cette présence, rappelée tous les ans ${ }^{13}$ ). Les enchères se font «au plus offrant et dernier enchérisseur à la chandelle éteinte». L'adjudication est double: il y a un adjudicataire pour la viande de boucherie et un autre pour la volaille. Le boucher qui emporte la ferme de la chair a seul l'autorisation de vendre de la viande pendant le Carême; en échange de ce monopole, il est chargé de livrer la viande nécessaire aux malades de l'Hôtel-Dieu

7 Archives Municipales de Lyon (AML), 6Fi00900: Affiches. Mandement de Monseigneur l'Archevêque pour l'usage de la viande pendant quelques jours du Carême prochain (31 janvier 1709).

8 Archives des Hospices civils de Lyon (AHCL), Hôpital de la Charité ou Aumône générale, E 35 : Administration de l'établissement. Délibérations du Bureau de l'Aumône générale (1629).

9 AML, 6Fi00900: op. cit. (31 janvier 1709).

10 Le reste de l'année, les règlements des bouchers et des poulaillers leur font explicitement défense de vendre de la viande «les jours et heures prohibez par l'Église», soit les dimanches et fêtes solennelles (HH 19: Règlements. Bouchers, 23 août 1668, article 15 et 30 mars 1700, article 16; HH 180: Règlements. Poulaillers et rôtisseurs, 11 septembre 1688).

11 AML, BB: Délibérations consulaires.

12 Archives Départementales du Rhône (ADR), BP 3624: Sénéchaussée. Ordre public. Corporations: bouchers et poulaillers de Carême ( $1^{\text {er }}$ février 1673 ).

13 Garden (1967). 
et de la Charité tout au long de l'année - temps du Carême et temps du charnage - à un prix fixé et probablement inférieur à celui du marché ${ }^{14}$. La viande fournie doit être de bonne qualité, "aultrement et à faulte de ce faire permis à MM. les recteurs d'en faire prendre ailleurs » à ses frais et dépens. Le fermier a l'obligation de régler le montant de sa ferme par moitié à l'Hôtel-Dieu et à l'Aumône générale avant Pâques. Il doit pour cela fournir «bonne et suffisante caution et certificateur». Les mêmes conditions s'appliquent dans le cas du poulailler de Carême, chargé d'approvisionner en cufs l'Hôtel-Dieu, là encore à prix fixé ${ }^{15}$. La permission de consommer de la viande pendant le Carême étant accordée au cas par cas par l'archevêque ou par ses vicaires à ceux qui, pour des raisons d'âge ou de santé, ne peuvent faire maigre ${ }^{16}$, le fermier de la chair est tenu d'exiger des malades et autres personnes dispensées des billets d'autorisation, à charge pour lui de les conserver en cas de contrôle.

Globalement, entre le milieu du XVII ${ }^{\text {ène }}$ siècle et 1700 , le montant annuel de la ferme varie, pour ce qui est de la viande de boucherie, entre 2500 et 3500 livres - à l'exception des années 1694 à 1696 au cours desquelles il chute à moins de 1500 livres ${ }^{17}$. Entre 1700 et 1714 il ne descend plus que trois fois en-dessous des 3500 livres et peut aller jusqu'à atteindre 5200 livres. Il est donc facile d'imaginer que seuls les bouchers aisés puissent se porter adjudicataires de la ferme, puisqu'à son coût propre s'ajoute l'engagement de garantir tout au long de l'année et à bas prix un approvisionnement suffisant aux deux hôpitaux, donc de disposer à la fois d'un solide réseau de fournisseurs et des fonds nécessaires à de tels investissements. Les bouchers susceptibles de pouvoir participer aux enchères sont donc peu nombreux ils sont en général seulement trois ou quatre à y prendre part - et ce sont souvent les mêmes qui, d'une année sur l'autre, se retrouvent adjudicataires. Entre 1677 et 1714 par exemple, soit sur une période de 38 années, 11 bouchers seulement se partagent la ferme, parmi lesquels deux endossent huit fois le rôle de fermier de la chair. L' «origine» de ces fermiers est également révélatrice de leur niveau de richesse, puisqu'ils tiennent tous boutique - à une exception près - dans les deux boucheries des Terreaux et de l'Hôtel-Dieu. Sans doute les bouchers des quartiers de Saint-Paul et Saint-Georges, plus pauvres, ne disposent-ils pas des fonds nécessaires pour pouvoir se porter enchérisseurs. Pour ce qui est de la volaille, les variations du montant de la ferme au cours des années 1658 à 1714 sont à la fois plus amples et plus irrégulières que pour la viande de boucherie, mais celui-ci reste compris entre 500 et 1500 livres. Entre 1677 et 1714, douze poulaillers se partagent le privilège d'exercer leur négoce pendant le Carême, le record étant détenu par Jacques Chanoine, fermier à treize reprises.

Ce système d'adjudication représente pour les deux hôpitaux de la ville une ressource financière non négligeable, ce qui explique qu'en 1709 comme en 1629 - les deux années où la ferme fut suspendue - l'autorisation accordée aux bouchers de la

14 Abad (1999, p. 242).

15 AHCL, Hôpital de la Charité ou Aumône générale, E 35: Administration de l'établissement. Délibérations du Bureau de l'Aumône générale.

16 ADR, BP 3624 (1 $1^{\text {er }}$ février 1673).

17 Pour tout ce qui concerne les procédures d'attribution de la ferme et le montant de celle-ci, voir les registres annuels de délibérations du bureau de l'Aumône générale (AHCL, Hôpital de la Charité ou Aumône générale, série $\mathrm{E}$ ). 
ville de vendre de la viande pendant le Carême se doubla d'un appel à l'aumône, destinée à compenser la perte subie par les deux établissements. En dehors de ces circonstances exceptionnelles, il est strictement défendu à tous les bouchers et poulaillers autres que les adjudicataires de la ferme de vendre de la viande pendant le Carême. Il s'agit par ces défenses à la fois de veiller au respect des prescriptions religieuses et de préserver le privilège financier des deux hôpitaux. C'est le pouvoir civil - bras séculier de l'Église - qui se charge de les faire connaître et appliquer. Tous les ans sont ainsi publiés et affichés par le présidial - tribunal d'appel uni à la sénéchaussée de Lyon - les noms des adjudicataires et les défenses faites à tous les autres bouchers et poulaillers de la ville, des faubourgs et des lieux alentour (à deux lieues à la ronde) et à toute autre personne de tuer des animaux et vendre de la viande, de la volaille et des cufs pendant le Carême. Les contrevenants encourent une amende et la confiscation de leur marchandise au profit des hôpitaux et des adjudicataires; la peine est augmentée en cas de récidive ${ }^{18}$. Seuls les bourgeois et citoyens de la ville détiennent le droit d'y faire entrer les chevreaux, les agneaux, la volaille et les œufs provenant de leurs métairies ou «maisons des champs».

Afin d'assurer le respect de ces défenses, les soldats et commis aux portes sont chargés de contrôler les personnes et les marchandises entrant en ville. Ils sont secondés dans cette tâche par des gardes improvisés - ouvriers en soie, affaneurs ou encore bouchers désœuvrés par force pendant le Carême et qui trouvent là un revenu d'appoint - directement employés pendant cette période par le fermier de la chair ou de la volaille. Ce dernier se voit par ailleurs autorisé à effectuer des visites de contrôle dans les maisons et boutiques de la ville, des faubourgs et des bourgs environnants dans le rayon des deux lieues prévues par les ordonnances. Il lui est en revanche interdit, comme à ses commis, de porter l'épée lors de ses recherches, à moins qu'il ne se fasse accompagner par des officiers de justice. Cette dernière mesure, qui permet aux autorités judiciaires de garder un oil sur le contrôle des infractions - puisque, dépourvus de la force concrète aussi bien que symbolique de l'épée, les fermiers se trouveraient bien démunis en cas de rébellion-, vise peut-être aussi à éviter, lors de telles perquisitions, des règlements de comptes anarchiques et sanglants entre bouchers ou poulaillers. Claude Vaginey, fermier de la chair qui se plaint en 1667 de ce que «la plupart des contrevenants font reffus de faire ouverture des endroits ou ils ont ladite viande de boucherie» et demande en conséquence «qu'il luy soit permis de faire faire ouverture desdits endroits par le premier serrurier requis », se voit accorder satisfaction à ses risques et périls ${ }^{1}{ }^{9}$. Rien de surprenant dans un tel contexte à ce que les visites soient plus souvent effectuées par des officiers de police ou de justice - sergents royaux ou huissiers audienciers assistés d'archers - que par les fermiers eux-mêmes.

\section{ESPACES, ACTEURS ET ENJEUX AUTOUR DU MARCHÉ PARALLELLE DE LA VIANDE PENDANT LE CARÊME}

L'attention portée par les autorités civiles à la manière dont doivent se dérouler les visites de contrôle, la plainte de Claude Vaginey - comme celles d'autres fer-

18 Voir par exemple ADR, BP 3624 ( $^{\mathrm{er}}$ février 1663 ).

19 ADR, 12G457: Chapitre de Saint-Just. Surveillance des corporations. Bouchers (6 février 1667). 
miers - laissent deviner que tous les Lyonnais ne respectent pas les défenses formulées dans les ordonnances de Carême et dévoilent en elles-mêmes l'existence pendant cette période d'un marché parallèle de la viande. Comme d'autres formes d'économie informelle, celui-ci a laissé sa marque dans les archives judiciaires à travers la série de plaintes, procès-verbaux de visites, interrogatoires, informations et - hélas beaucoup plus rarement - jugements conservés pour l'essentiel dans les archives du tribunal de la sénéchaussée ${ }^{20}$. La nature même du phénomène, par définition clandestin, comme le mode de production des sources permettant de l'appréhender - les procès-verbaux sont dressés essentiellement à la suite de plaintes ou de dénonciations et laissent donc dans l'ombre des pans entiers de ce marché noir rendent évidemment hasardeuse, si ce n'est impossible, toute entreprise de quantification. Sur les 82 cas d'infraction à la règle relevés entre 1658 - date de la première affaire conservée - et 1714 , les plus nombreux concernent par exemple les premières années du XVIII ${ }^{\text {ème }}$ siècle: rien ne permet de savoir si cela reflète réellement une amplification du marché noir - et donc des pratiques de contournement de la norme - au cours de ces quinze ans, un durcissement du contrôle et de la répression - par le recours plus systématique à la justice - d'un phénomène globalement stable, ou tout simplement une meilleure conservation des sources, ou enfin s'il y a combinaison de ces trois facteurs d'explication. Impossible à quantifier, le phénomène du marché noir de la viande pendant le Carême peut néanmoins être appréhendé de façon qualitative, à travers ses manières de fonctionner dans l'espace urbain, ses acteurs, ses enjeux enfin.

Le dépouillement des procès-verbaux de visites nous emmène sur les pas des sergents et des huissiers jusqu' aux «tueries », aux entrepôts et aux boutiques clandestines. Il permet d'esquisser une géographie de l'illicite et met en lumière le rôle central des faubourgs dans l'inscription spatiale de ce marché parallèle.

Pour ceux qui prennent le risque d'exercer un tel commerce au sein des murs de la ville, la plus grande prudence est de mise. Les bouchers et poulaillers qui poursuivent ainsi leurs activités pendant le Carême choisissent pour s'y livrer leur arrière-boutique, dans un souci de plus grande discrétion. Un tel trafic peut aussi s'abriter dans d'autres boutiques - celle par exemple d'un chandelier, d'un marchand de vin, d'un écrivain ou encore d'un marchand toilier - ou dans les maisons privées. Les demeures des aristocrates et des bourgeois forment des cachettes particulièrement appréciées car le statut de leurs propriétaires leur permet de s'opposer plus facilement aux visites de contrôle. Il est par ailleurs à la fois aisé et tentant pour ces catégories sociales de franchir la frontière entre le légal et l'illégal en outrepassant le privilège qui leur permet de faire entrer à Lyon les chevreaux et les agneaux provenant de leurs «maisons des champs ${ }^{21}$. Cet état de fait incite Jean Paradis, fermier de la chair en 1668, à former une plainte contre ceux qui cachent de la viande dans la ville «et choisissent mesme pour leur servir de retraite les maisons des personnes de condition ou d'autres, l'accès et l'entrée desquelles est difficilement permis». Il requiert qu'il lui soit permis de «faire visiter les carrosses entrans dans la ville ou il croira y pouvoir avoir de la viande $\gg^{22}$. Le commerce d'animaux ou

$20 \quad$ ADR, BP 3624

21 ADR, BP 3624 (8 mars 1668).

22 ADR, BP 3624 ( 3 mars 1668). 
de viande de boucherie constitue par ailleurs une activité lucrative comme une autre et certains de ces «bourgeois ${ }^{23}{ }^{2}$ 'hésitent pas à s'y livrer, y compris pendant le Carême. En 1702, c'est une véritable boucherie clandestine qui est ainsi découverte dans le domaine d'un nommé Rivoyre, bourgeois du quartier Saint-Thomas de Fourvière ${ }^{24}$.

Les hôteliers et aubergistes de la ville prennent eux aussi à l'occasion le risque de faire commerce illicite de viande, de servir des repas gras ${ }^{25}$ ou d'en accommoder pour des personnes de qualité ${ }^{26}$. Toutes ces pratiques constituent autant de contraventions aux ordonnances «qui deffendent aux hostelliers, maistres d'auberges et autres personnes qui donnent à manger de fournir de la viande les jours que l'esglize en deffend l'usage $\gg^{27}$. La viande trouvée chez la veuve Masson, qui tient auberge rue de l'Angile, était ainsi destinée à être vendue, « ou peut estre ce qui seroit encore plus criminel [à être donnée] à manger chez elle a des jeunes gens debauchez ${ }^{28}$. Le scandale est encore plus grand quand ces repas gras s'accompagnent de jeux ${ }^{29}$, révélant l'existence, autour de la vie nocturne et particulièrement des sociabilités liées à la table, de rituels sociaux qui entrent en compétition avec ceux de l'Église.

La principale difficulté demeure dans tous les cas de parvenir à faire entrer à Lyon la viande ou les animaux de contrebande. Le passage des portes - en théorie bien gardées - reste un obstacle majeur, comme en témoigne le nombre de saisies réalisées à ce moment-là, même si les fraudeurs rivalisent de ruses: lorsque la marchandise n'est pas simplement camouflée dans un linge ou un tablier, sous un justaucorps ou un manteau, ou encore cachée dans des sacs sous d'autres denrées - le chargement de pommes d'un âne, par exemple ${ }^{30}$-, elle est confiée à des tiers que leur profession rend moins soupçonnables ou à des femmes, dûment et discrètement « escortées », dont on sait qu'elles seront sans doute moins sévèrement punies en cas d'arrestation. Pour qui dispose d'une embarcation, le Rhône et la Saône constituent, particulièrement la nuit, d'autres voies possibles de contrebande. L'ordonnance du présidial qui définit chaque année les modalités du commerce de la chair pendant le Carême fait d'ailleurs explicitement défense à tous les bateliers et à toute autre personne de faire entrer dans la ville par les rivières de la viande, de la volaille et des œufs, à peine d'amende et de confiscation des bateaux ${ }^{31}$, ce qui montre assez que les voies d'eau, moins faciles à contrôler, sont largement utilisées par les fraudeurs ${ }^{32}$. Des bouchers de Vaise, la Croix-Rousse et la Guillotière acheminent ainsi nuitamment par la rivière des sacs de viande qu'ils font ensuite passer discrètement par dessus les murailles de la ville ${ }^{33}$.

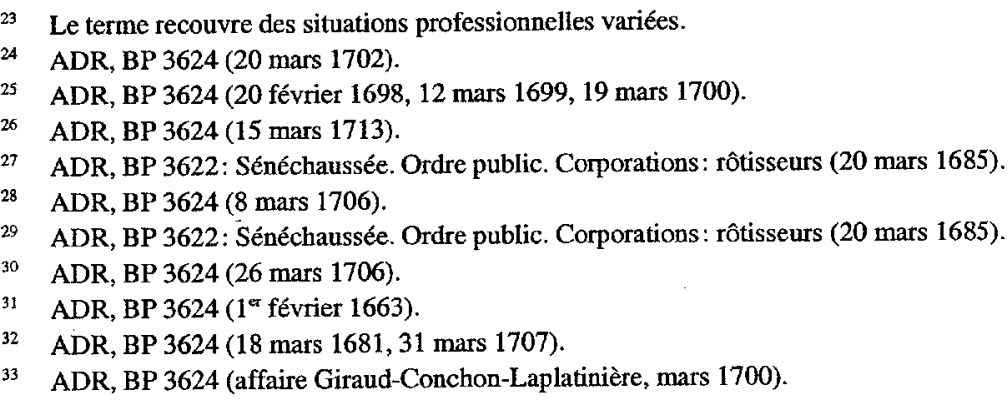


Ce dernier exemple laisse deviner la place centrale qu'occupent les faubourgs et les bourgs voisins de Lyon dans cette géographie de l'illicite. En théorie, la réglementation ne limite pas en effet la portée des interdictions à la ville intra muros, mais en prévoit l'extension à un rayon de deux lieues autour de Lyon. Dans cette zone, tout commerce de chair est interdit pendant le Carême afin précisément d'assurer l'approvisionnement des fermiers en viande et en volaille et de les prémunir de la concurrence. Jean-François Castorisan, fermier de la chair, explique ainsi en 1699 qu'il ne se serait pas porté adjudicataire de la ferme «s'il avoit esté permis d'establir des boucheries a deux lieues a la ronde de cette ville puisque cela empescheroit non seulement qu'il ne peut pas avoir des bestiaux, mais encore le detruiroit entierement et luy osteroit le moyen de payer le prix de sa ferme et les charges qu'il est obligé de supporter; car il seroit fort facile aux bouchers de campagne qui s'etabliroient a deux lieues a la ronde de donner la viande a vil prix puisqu'ils auroient beaucoup plus de facillité de l'avoir que le fermier du Caresme, et ne supporteroient aucun droit comme ceux d'entrée de la ville et de la ferme, en sorte que si cela avoit lieu jamais aucune personne ne voudroit encherir ny prendre ladite ferme, et par ce moyen les pauvres des deux hopitaux se trouveroient privés du bénéfice qu'ils en tirent annuellement ${ }^{34}$.

Or quelques bouchers et hôteliers des faubourgs profitent justement des avantages liés à leur implantation hors les murs - facilités d'approvisionnement, décharge des droits d'octroi, risques moins grands encourus face aux contrôles pour poursuivre leur activité durant le temps du Carême et ce parfois au vu et au su de tous. Certains bouchers de Vaise, la Croix-Rousse et la Guillotière tiennent ainsi impunément «boutique ouverte» où viennent s'approvisionner, selon des témoins, «quantité de personnes» de la ville ${ }^{35}$. Quant aux hôteliers de la Croix-Rousse et de Caluire, ils jouent de leur situation au débouché des chemins de Bress ${ }^{36}$ pour se livrer à de véritables actes de brigandage, enlevant de force aux voituriers les volailles qu'ils apportent pour les poulaillers de Carême, en les payant «comme il leur [plaît], ce qui degarny non seulement la boutique des supliants mais empeschent encor aux voituriers de venir en cette ville attendu qu'ils n'ont plus la liberté de tirer de leur volaille son prix $»^{37}$.

Un tel phénomène appelle des éléments d'explication et ramène au statut juridique ambigu des bourgs les plus proches de la ville. Si les faubourgs de Lyon dépendent en effet de la circonscription du présidial, Vaise, la Croix-Rousse et la Guillotière sont - malgré leur assimilation fréquente à des faubourgs - de véritables bourgs. Le cas de la Guillotière, situce sur la rive gauche du Rhône, est particulièrement complexe puisque le consulat de Lyon exerce la justice sur cette terre pourtant dauphinoise. Rien n'interdit de penser que les contrevenants jouent précisément sur l'ambiguitté de ces statuts juridiques pour enfreindre la loi avec plus de liberté. Pour ce qui est des villages plus éloignés, mais cependant toujours situés dans cette zone des deux lieues (Oulins, Francheville, Fontaine, Caluire par exemple), la difficulté du contrôle est renforcée par d'éventuels conflits de juridiction mettant aux prises le

\footnotetext{
34 ADR, BP 3624 (procédure Castorisan-Aumône générale, avril 1699).

35 ADR, BP 3624 (affaire Giraud-Conchon-Laplatinière, mars 1700).

36 La région produit déjà à l'époque une bonne partie des volailles destinées à la consommation lyonnaise.
}

37 ADR, BP 3624 (11 mars 1711). 
présidial de Lyon et les seigneurs locaux. Dans le cas de Caluire, le présidial se heurte ainsi au seigneur haut justicier du lieu, en l'occurrence le chanoine et archidiacre de l'église royale et collégiale de l' ̂̂le-Barbe ${ }^{38}$. Ce dernier se plaint en 1699 d'une confiscation de viande opérée par le fermier Jean-François Castorisan chez un boucher de Caluire. Il argue que les fermiers ne peuvent faire valoir hors de la ville les privilèges qu'ils ont reçus des recteurs de la Charité, «ce qui est si connu que dans presque tous les fauxbourgs on vend publiquement de la viande pendant le Caresme $»^{39}$. Mais quand bien même «ils pourroient l'empescher dans lesdits fauxbourgs», il est selon lui «inoui et extraordinaire qu'ils le puissent à une lieue dedans les terres particulières des seigneurs ${ }^{40}$. Ces derniers sont en effet obligés par les règlements de police de veiller à ce que leurs justiciables, « qui a cause de leurs infirmités pourroient avoir besoin de la viande de caresme $»^{41}$, puissent en trouver sur les lieux. Ils ont en outre «des charges tres considerables a suporter comme la nourriture et soulagement des pauvres ${ }^{42}$. Or «les maisons de la Charité et de l'Hospital n'estant principallement establies et ne servant en effet ordinairement que pour les pauvres de la ville, les privileges qui leur sont accordés par cette consideration [c'est-à-dire le monopole de la vente de la chair en Carême] ne doivent point estre etendus au dehors, ny estre a la charge de ceux qui ne resident point dans cette ville $»^{43}$. Il serait au surplus «bien rude et bien extraordinaire » de vouloir obliger ces derniers à venir acheter de la viande à Lyon «le triple plus qu'elle ne leur cousteroit sur les lieux $»^{44}$. Bien que tous ces arguments apparaissent comme parfaitement recevables, le procureur du roi conclut pourtant l'affaire en faveur de Castorisan et réaffirme au passage la préeminence de la justice présidiale sur la justice seigneuriale. Il note en effet que «quoy qu'on ne puisse pas contester au sieur archidiacre la justice haute moyenne et basse dans l'estendue de Caluire (...), neanmoins cette qualité de seigneur haut justicier ne le dispense pas de l'observation des ordonnances générales de police qui deffendent l'exposition de la viande en caresme, parce qu'il repugne qu'on puisse distribuer publiquement ce qui n'est pas permis de vendre en secret ${ }^{45}$. Rien ne dit pour autant que, malgré de telles affirmations d'autorité, des pratiques illicites ne continuent de fleurir sur le terreau formé par les vides juridiques ou les chevauchements d'influences.

Il est évidemment extrêmement difficile d'estimer le nombre de Lyonnais concernés par ce marché parallèle. Les quantités de viande saisies sont, lorsqu'elles sont mentionnées, d'importance très variable - de quelques livres à plusieurs animaux entiers - et pourraient suivant les cas nourrir aussi bien un simple ménage que tout un quartier. Impossible à quantifier, la demande à l'égard du marché clandestin n'en est pas moins présente, qu'elle émane d'ailleurs des consommateurs ou des agents de ce commerce. Pour ce qui est des premiers, le champ des interrogations reste là encore très ouvert. S'agit-il de personnes réellement concernées - pour

\footnotetext{
38 Il est seigneur haut justicier de Colonges, Bourg Saint-Rambert et Caluire.

ADR, BP 3624 (28 mars 1699).

Ibidem.

Ibidem.

Ibidem.

ADR, BP 3624 (8 février 1700).

Ibidem.

Ibidem.
} 
des raisons d'âge ou de santé - par les dispenses de jeûne, d'individus bien portants peu soucieux de respecter les interdits religieux ${ }^{46}$ - à l'exemple (extrême) de cet abbé qui se fait accommoder de la volaille chez un poulailler ${ }^{47}$ - ou encore de protestants ${ }^{48}$ qui par définition échappent à ces restrictions alimentaires? Il ne faut pas écarter par ailleurs l'hypothèse d'une consommation de viande - après 1685 et la révocation de l'Édit de Nantes - chez les nouveaux convertis au catholicisme qui resteraient secrètement attachés aux usages de leur ancienne confession. Du côté des bourgeois et des aristocrates, la transgression des interdits pourrait signaler l'ébauche d'une diffusion - par le haut de la société - de nouveaux modèles de consommation et d'un rapport plus libre à la religion. Sont ainsi saisis chez un certain Paul Solicoffre, bourgeois, six moutons «qu'il destinoit a en nourrir son mesnage pendant le caresme ${ }^{49}$. Reste que le manque de données ${ }^{50}$ ne permet pas de se livrer à une catégorisation fine des consommateurs. La justice s'arrête finalement bien souvent au seuil des foyers, laissant dans l'ombre les pratiques privées, le rapport intime que les individus entretiennent avec les lois divines et humaines. Elle recherche avant tout les agents du marché noir, et non les simples consommateurs de viande, ce qui explique que le profil et les intérêts des premiers nous apparaissent de manière beaucoup plus claire.

La vente de volaille pendant le Carême peut ainsi constituer, pour des artisans modestes ou des femmes pauvres (par exemple des revendeuses de fruits et légumes), une activité et donc un revenu complémentaires ${ }^{51}$. Poursuivre, malgré les interdictions, leur activité pendant cette période peut aussi représenter pour les bouchers et les poulaillers les plus pauvres, que l'arrêt total de leur négoce pendant quarante jours mènerait à la faillite, une véritable «stratégie de survie». Lorsqu'ils sont arrêtés et reconnaissent leur délit, ce sont des raisons d'ordre économique qu'ils invoquent pour justifier leur geste. Le poulailler Martin Durozier convient ainsi lors de son interrogatoire que l'on trouva chez lui, rue de la Fronde, de la volaille et «demeure d'accord qu'estant dans un estat malheureux ne scachant comment gagner sa vie il avoit acheté ladite volaille et gibier pour les revendre et tacher d'en tirer un mediocre proffit pour aider a nourrir sa femme qui est indisposée et deux enfans qu'il a en bas age, n'ayant point d'autre profession que celle de poulailler, laquelle il ne peut pas mesme exercer ni tenir boutique ouverte comme font les autres poulaillers attendu sa pauvreté, et cela est sy vrai qu'il avoit mesme emporté un louis d'or d'une personne charitable pour achepter la volaille et gibier qu'on luy a saisy, estant les seules pieces de volaille et gibier qu'il ayt eu pendant ce caresme chez luy ${ }^{52}$. Le discours est attendu, ressassé au fil des affaires et dénote une inté-

46 Sur les liens entre consommation de viande en Carême et phénomène de déchristianisation, voir Abad (1999).

47 ADR, BP 3624 (19 février 1687).

48 D'après l'État général des familles protestantes de la ville, dressé par les anciens à l'intention du synode de Bussy, 248 familles protestantes vivent à Lyon au milieu du XVIr ${ }^{\text {me }}$ siècle, in Martin (1986).

49 ADR, BP 3624 (8 mars 1668).

so Cinq artisans, deux bourgeois, un praticien, un avocat en parlement, les trois fils d'un voyer de ville composent le maigre échantillon des personnes chez qui ou sur qui a été saisie de la viande susceptible de n'être destinée qu'à un usage strictement privé.

51 ADR, BP 3624 (23 mars 1703, 2 avril 1711).

52 ADR, BP 3624 (21 mars 1706). 
riorisation certaine de la norme: le coupable joue sur l'argument de la pauvreté réelle ou prétendue - dont il sait qu'il lui évitera une trop lourde peine. Derrière la rhétorique émerge cependant une réalité incontestable, celle d'une grande partie de la population d'Ancien Régime qu'un rien peut suffire à faire glisser dans la pauvreté et qui est ainsi contrainte de jouer sans arrêt aux marges du légal et de l'illégal. Dans le cas précis du Carême, les contraintes sont telles - absence totale de revenus pendant quarante jours - que la seule solution envisageable pour certains bouchers qui veulent échapper à la paupérisation réside dans la transgression ${ }^{53}$. La mention du louis d'or, obtenu d'une «personne charitable», permet d'introduire un dernier élément. Deux interprétations s'offrent à nous: soit cette pièce est le fruit d'un pur acte de charité qui a permis à ce poulailler d'acheter de la volaille pour la revendre ensuite clandestinement à son domicile; soit Martin Durozier joue le rôle de courtier pour des clients aisés (et peu soucieux de respecter les interdits du Carême), hypothèse qui peut être corroborée par le fait que lui-même se dise dans l'incapacité financière de tenir boutique. Dans les deux cas, cet exemple montre aussi que les acteurs ne sont pas forcément complètement démunis face à la norme et qu'ils arrivent au contraire à créer «des formes de solidarités intégratrices» à travers «des systèmes d'entraide dont ils fixent eux-mêmes les règles du jeu ${ }^{54}$.

Pour les bouchers et poulaillers plus aisés enfin - qui sont parfois d'anciens adjudicataires surpris en flagrant délit d'infraction aux règlements ${ }^{55}$, la période du Carême offre des occasions de s'enrichir en vendant la viande à meilleur marché que le fermier lui-même. Jean-François Castorisan se plaint ainsi en 1700 de trois bouchers concurrents qui lui ont porté «un préjudice très considérable, non seulement parce qu'ils lui ont fait acheter la viande plus chère et se sont tenus sur les avenues pour l'accaparer mais encore parce qu'ils la donnaient à meilleur marché " ${ }^{56}$; il estime ses dommages à plus de 3000 livres de perte et réclame 2000 livres de dommages et intérêts (il en obtiendra 60 livres!) ${ }^{57}$. Ce procédé de « concurrence sauvage contre un monopole établi de droit ${ }^{58}$ est parfois encouragé involontairement par le fermier en personne lorsque ce dernier, contraint de réaliser en six semaines un bénéfice considérable afin de pouvoir régler le montant de sa ferme et dégager en sus une marge pour son propre profit - le tout en période de sous-consommation ${ }^{59}-$, ne respecte pas les «prix plancher» auxquels il s'est engagé à vendre sa viande, allant parfois jusqu'à «donner de mauvaise viande a ceux qui ne sont pas en état de la luy payer selon son intention $»^{60}$. Comme le laissait envisager la plainte formulée cidessus par Jean-François Castorisan, une telle concurrence peut causer au fermier chargé «de payer une grosse ferme et faire des grands frais pour l'entretien des officiers ou commis particuliers pour y veiller ${ }^{61}$-des pertes financières considérables.

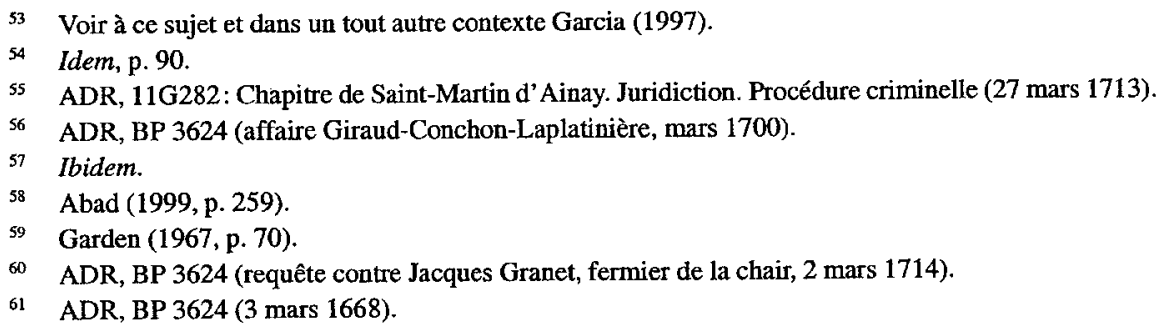




\section{DISCOURS ET NÉGOCIATIONS AUTOUR DE LA NORME}

Les normes qui régissent le commerce de la viande pendant le Carême sont donc régulièrement contournées - parfois même avec la complicité involontaire des fermiers - par une partie de la population qui trouve dans les pratiques illicites décrites plus haut des modes de survie ou d'enrichissement. Pour autant, les comportements de ces acteurs sont loin de s'opposer mécaniquement aux contraintes formelles énoncées par l'Église et relayées par le pouvoir civil. En ce sens, les rapports entre normes et pratiques ne peuvent être appréhendés uniquement en termes de déviance: contournée, la norme fait aussi l'objet de stratégies manipulatrices; dans le même temps, de nouvelles formes de droit peuvent naître des pratiques et des comportements ${ }^{62}$. Les arguments développés par le seigneur de Caluire montraient déjà en un sens comment la réglementation pouvait être discutée, voire réinterprétée - avec de solides arguments à l'appui - dans un sens imprévu. La documentation fournie par les sources judiciaires permet d'élargir cette approche et d'appréhender la dynamique des échanges - renégociés en permanence - entre normes et comportements. Gestes et discours font en effet apparaître l'éventail des champs dans lesquels s'inscrivent les rapports entre les individus et la règle, et qui font que cette dernière est en pratique constamment redéfinie par les usages eux-mêmes.

La fraude est rarement le fait d'individus isolés et, lorsque ses acteurs sont pris sur le fait, le premier degré de réaction face aux représentants de la loi reste - si l'on en croit les procès-verbaux de contravention - la violence, physique ou verbale. Lorsqu'il s'agit de contrôles aux portes de la ville, il n'est pas rare que les contrevenants s'octroient le passage par la force ${ }^{63}$, selon des techniques quelquefois assez élaborées: quelques hommes se chargent par exemple de «neutraliser» les gardes tandis qu'une ou deux femmes franchissent pendant ce temps les portes avec leur marchandise ${ }^{64}$. Au delà de la brutalité apparente de la tactique, cette utilisation des femmes - déjà évoquée plus haut -, qui courent un risque moins grand face à la justice, révèle de la part des contrevenants à la fois une connaissance certaine de la loi et une exploitation de la réalité judiciaire. Ces derniers jouent, pourrait-on dire, sur le hiatus existant entre la fiction d'une loi théoriquement égale pour tous et sa réalité, ce qui ramène plus globalement à toute la culture juridique d'Ancien Régime qui veut qu'une même loi ne soit dans les faits jamais la même selon les individus et les circonstances auxquels elle s'applique.

Lors des contrôles à domicile, le fermier ou l'officier se heurte souvent à porte close et se voit contraint de recourir à un serrurier pour l'ouverture des endroits soupçonnés de receler de la viande. Contraint par la procédure de se faire assister de deux témoins lors de ses visites, il est presque toujours confronté au refus des voisins sollicités en ce sens ${ }^{65}$. Cette résistance passive et quasi-systématique opposée par la population aux représentants de la loi se mue aussi à l'occasion en

62 Sur la question des interactions entre normes et pratiques, envisagée sous un jour nouveau au milieu des années 1980, voir concernant l'époque moderne: Levi (1985), Quaderni Storici (1986), Raggio (1990), Kuhen (1991), Cerutti (1995) et Schlumbohm (1997).

${ }^{63}$ ADR, BP 3624 (10 avril 1680, 17 février 1698).

64 ADR, BP 3624 (affaire Cartier, 3 mars 1700).

6s ADR, BP 3624 (26 février 1684, 20 mars 1688). 
une opposition violente suscitée par les cris et les appels au secours du contrevenant lui-même: «rébellion ${ }^{66}$, «caballe ${ }^{67}$, «émotion populaire ${ }^{68}$ sont des termes qui reviennent fréquemment dans les rapports des huissiers, contraints de se retirer bredouilles ou de recourir en urgence aux soldats du guet.

Sont aussi révélés, à travers ces formes de résistance plus ou moins violente aux agents de la loi, les réseaux de solidarité à l'échelle du quartier. Le jeu ne se fait plus ici sur la connaissance de la norme mais plus prosaïquement sur la maîtrise des rapports de force au sein de la cité. Il est d'ailleurs possible de se demander si les solidarités qui s'expriment alors ne sortent pas renforcées de ces épisodes de violence et si finalement ces manifestations quasi-rituelles d'opposition à l'application de la norme ne sont pas en elles-mêmes et tout à la fois révélatrices et créatrices de connivences. Lorsque les personnes surprises en contravention sont des bouchers ou des poulaillers, les violences exercées sur la personne du fermier peuvent en outre être interprétées comme étant l'expression de tensions sociales, de rancœurs de la part des «petits» à l'égard du «gros» qui a pu s'octroyer - peut-être de manière frauduleuse - le monopole de la ferme. Les gestes se chargent alors d'une force symbolique: le boucher Bernard et ses enfants enlèvent ainsi par la force à Jean-François Castorisan «son chapeau, sa perruque et son manchon, en sorte qu'il [est] obligé de s'en retourner chez luy la teste nue ${ }^{69}$. D' autres, surpris avec de la viande au passage du pont du Rhône, préfèrent jeter celle-ci dans le fleuve pour éviter qu'elle soit saisie, disant «qu'ils aimoient mieux la jetter que si [le fermier] en profitoit $»^{70}$. Une telle réaction se situe bien au delà du réflexe instinctif consistant à se débarrasser de l'objet du délit et le geste est ici largement explicité : il exprime clairement le ressentiment à l'égard du fermier que le Carême enrichit tandis que les petits bouchers peinent à survivre pendant ces quarante jours. En ce sens, les différentes réactions d'insoumission et de violence rapportées par les procès-verbaux ne semblent pas avoir pour motivation profonde la défense de droits traditionnels ${ }^{71}$, mais plutôt constituer des réponses, sans doute moins spontanées et plus réfléchies qu'il n'y paraît, à l'enrichissement - voire à la corruption - d'une minorité. Par ailleurs, les atteintes physiques commises sur les personnes du fermier ou de ses commis renvoient à l'interprétation que fait W. Beik des émeutes antifiscales du XVII ${ }^{\mathrm{eme}}$ siècle: ces violences ne seraient pas seulement une manière de soulager une injustice perçue par la communauté mais aussi, au travers des atteintes physiques symboliquement infligées aux agents du fisc, l'expression d'une «culture du châtiment» («culture of retribution») ${ }^{72}$.

66 ADR, BP 3624 (15 mars 1688, 11 avril 1696, 28 février 1700, 19 mars 1700, $1^{\text {er }}$ avril 1700).

67 ADR, BP $3624\left(1^{\text {er }}\right.$ avril 1700$)$.

68 ADR, BP 3624 (28 février 1706, mars 1706).

69 ADR, BP 3624 (11 avril 1696).

70 ADR, BP 3624 (22 mars 1688).

71 Ce qu'E.P. Thompson a montré à l'inverse dans un contexte bien différent, celui des émeutes frumentaires dans l'Angleterre du XVIII İme siècle: ces révoltes sont sons-tendues selon lui par un sentiment de légitimité de la part du peuple, légitimité qu'il définit de la sorte: "by the notion of legitimation I mean that the men and women in the crowd were informed by the belief that they were defending traditional rights or customs; and, in general, that they were supported by the wider consensus of the community » ( par la notion de légitimité, $j$ ' entends que les hommes et les femmes de ces foules étaient guidés par la certitude de défendre des droits et des coutumes traditionnels, et qu'en général ils avaient le plus large soutien de la communauté»), Thompson (1971,p. 78) et (1988, p. 33) pour la traduction française. 
Menaces, blasphèmes et insultes - dont la plus fréquente est de loin celle de voleur, ce qui est aussi une façon de renverser la situation et l'accusation -, s'accompagnent d'ailleurs de tout un discours de mépris à l'encontre de la loi et de ses représentants: "nous nous foutons de votre ordonnance ainsy que de ceux qui l'ont rendue $»^{73}$, «je me moque tant de vous autres que du fermier et de la justice ${ }^{74}$. Tous ces discours peuvent être interprétés comme l'expression d'une certaine liberté de fait par rapport à la rigidité apparente de la loi, liberté accentuée par la difficulté pratique de faire respecter cette loi. Ils trahissent aussi probablement, dans le cas de saisies mineures, le sentiment d'injustice du petit contrevenant, «attrapé» par malchance quand d'autres tiennent impunément boutique ouverte où ils vendent de la viande sans être inquiétés. Le discours de dénigrement de la loi et de ses représentants exprime alors toutes les rancœurs nées de ce hiatus - évoqué plus haut entre la fiction de la loi (théoriquement égale pour tous) et la réalité de son application.

Une fois dressé le procès-verbal de contravention, l'accusé se trouve soumis selon le principe de la procédure inquisitoire - à un interrogatoire, éventuellement complété en cas d'incohérences et de contradictions par l'audition de témoins information. Ce n'est qu'ensuite que le procureur du roi rend ses conclusions et qu'intervient le verdict. Ces interrogatoires, et particulièrement les arguments avancés par les inculpés, permettent de s'interroger sur les compétences que les individus font intervenir lors de leur confrontation à la justice ${ }^{75}$. La première constatation est que les discours des accusés apparaissent alors comme dépouillés de la part d'exagération verbale caractéristique du contexte de la plainte ou du procèsverbal - et dont il est difficile de savoir si elle n'est pas en partie due à une manouvre du rédacteur lui-même pour aggraver la culpabilité du contrevenant. Nul n'ignore par ailleurs qu'adopter une attitude coopérative pendant l'interrogatoire peut être un moyen facile d'échapper à une trop lourde peine.

Beaucoup d'accusés utilisent comme seule arme de défense la négation en bloc des faits qui leur sont reprochés ${ }^{76}$. Si l'argument renvoie à une stratégie de défense des moins élaborées, il révèle peut-être aussi - et ce dès la fin du XVII ${ }^{\text {rme }}$ siècle et les premières années du XVIII ${ }^{\text {ène }}$ siècle - ce que F. Freundlich a pu observer plus tard à Paris, à savoir «un sentiment d'affranchissement, la volonté de ne pas céder à l'intimidation ${ }^{77}$. À l'inverse, certains accusés - moins nombreux - tentent de légitimer l'infraction commise en se réfugiant derrière une prétendue ignorance des règlements ${ }^{78}$. Un hôtelier avoue ainsi avoir acheté de la volaille pour ses propres besoins et «n'estime pas qu'il soit deffendu a des particuliers d'en acheter pour leur usage indifféremment de toutes sortes de personnes $>^{79}$. D'autres tentent de prouver l'innocence de leurs intentions par le fait que la viande saisie chez eux «n'estoit

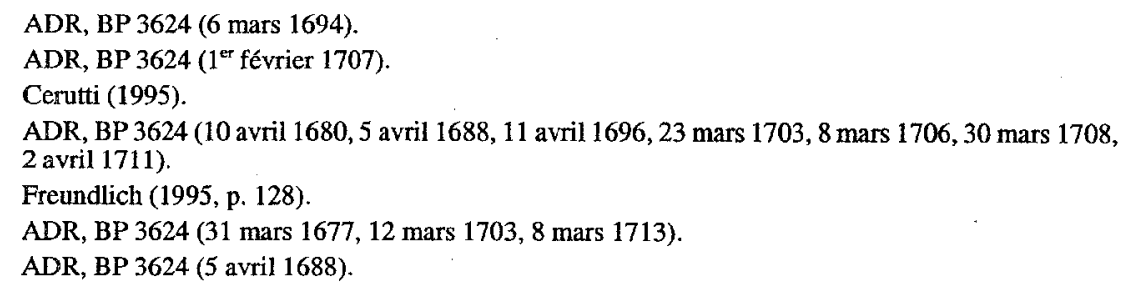


cachée ni fermée ${ }^{80}$ ou cherchent à limiter la portée du délit en affirmant que c'est la première fois qu'ils se livrent à une telle infraction ${ }^{81}$.

Les stratégies de légitimation diffèrent donc selon les individus et les outils intellectuels dont ceux-ci disposent face à la justice, à ses procédures et au domaine abstrait pour beaucoup - des normes écrites. La connaissance de la loi et de ses conditions concrètes d'application permet ainsi aux accusés de construire un premier système de défense. L'argument de la maladie ou de la vieillesse est employé à plusieurs reprises pour justifier la possession de viande ${ }^{82}$. Mais le subterfuge, qui renvoie par ailleurs à tout un discours scientifique sur la fonction thérapeutique de la nourriture ${ }^{83}$, ne réussit pas toujours. Il est ainsi répliqué à Antoine Bessenay, poulailler chez qui s'est trouvé du gibier dont il prétend qu'il était destiné à sa femme et à ses deux enfants malades, que les personnes «qui sont obligez de manger de la viande pour quelque indisposition prennent plutost de la viande de boucherie que des canards et des oyzeaux de riviere, soit parce qu'elle couste moins, soit parce qu'elles sont plus utiles pour la santé, ainsy sa reponse n'est qu'un déguisement $~^{84}$. Des bouchers des faubourgs utilisent quant à eux comme argument de défense une autre exception légale à la règle en se disant chargés de fournir de la viande aux troupes de passage ${ }^{85}$, ce qui les autoriserait à tenir boutique ouverte en vertu d'une «bonne permission» reçue de l'étapier. La connaissance précise des modalités concrètes d'application des règlements peut aussi être utilisée par les accusés dans leur confrontation avec les représentants de la loi ou les membres du tribunal. Antoine-François Marrel, un tripier qui tient dans sa boutique un commerce clandestin de volaille, utilise ainsi sa connaissance de la loi pour en refuser l'entrée au sergent royal à moins, selon les règlements, «qu'il ne luy montrast ses commissions et qu'il ne fust assisté de quelques voisins ${ }^{86}$. De la même façon, un ami venu témoigner au procès de Philibert Dufourt, surpris à la Guillotière avec de la volaille, reproche au fermier une fouille qu'il considère comme illégale, en arguant «que ce n'estoit pas dans le fauxbourg ou il falloit fouiller les gens et que c'estoit a la porte ${ }^{87}$.

Un second modèle de légitimation s'appuie quant à lui non plus sur la connaissance (ou la prétendue méconnaissance) de la loi mais sur la maîtrise par les inculpés des règles de la procédure judiciaire qui leur permet de réemployer à leur profit le système de la plainte, du témoignage ou de la preuve. Un bourgeois, accusé de tenir un commerce clandestin de viande dans sa «maison de plaisir» aux portes de Lyon, tente ainsi - vainement - de manipuler la justice en cherchant à faire établir par le propriétaire de la dite demeure un acte reconnaissant (contre la vérité) que «le louage [en] estoit finy depuis la saint Martin dernière ${ }^{88}$. Si quelques-uns se limitent

\footnotetext{
80 ADR, BP 3624 (23 mars 1703).

81 ADR, BP 3624 (22 mars 1684, 6 mars 1706,2 avril 1708).

82 ADR, BP 3624 (28 février 1684, 18 mars 1688, 5 avril 1688, 14 mars 1689, 11 mars 1693, 20 février 1698,28 février 1706,11 avril 1707,3 avril 1708).

83 Flandrin et Montanari (1996), Vigarello (1993).

84 ADR, BP 3624 (14 mars 1689).

85 ADR, BP 3624 (28 février 1700).

86 ADR, BP 3624 (28 février 1684).

87 ADR, BP 3624 (20 février 1698).

88 ADR, BP 3624 (30 mars 1703).
} 
par ailleurs lors des procès à essayer de prouver que la viande trouvée chez eux est le résultat d' «un tour que quelques personnes mal intentionnées [leur] ont voulu jouer ${ }^{89}$, d'autres renversent purement et simplement l'accusation en supposant ouvertement qu' « il ne seroit pas impossible que lesdits huissier ou assistans eussent mis eux mesmes ladite volaille dans [leur] grenier pour [les] faire tomber en contravention ${ }^{90}$ ou portent plainte - témoins à l'appui - contre le fermier et ses gens pour violences ou perquisition abusive ${ }^{91}$. Lorsque les parties se connaissent, de vieux conflits - réels ou inventés - resurgissent à l'occasion derrière ces plaintes et contreplaintes. Un hôtelier de la Croix-Rousse, qui paraît être l'un des fournisseurs habituels des fermiers, avait ainsi offert de vendre des poules au poulailler de Carême, mais celui-ci se trouva dans l'incapacité de les payer comptant et le marché fut rompu. C'est selon son témoignage «en haine de cela et d'une somme de 22 livres que les fermiers luy doivent en reste pour fourniture à eux faite pendant le caresme de l'année $1708 \mathrm{qu}$ 'ils suscittent au respondant l'affaire pour laquelle il est ceans ${ }^{92}$. Celle-ci met aussi en lumière le fonctionnement des réseaux d'approvisionnement au sein desquels les hôteliers des faubourgs jouent un rôle de pivot entre la ville et ses ceintures nourricières. Leur proximité avec la campagne leur permet en effet d'aller facilement se fournir en volaille dans les fermes des environs de Lyon, pour la revendre ensuite aux fermiers de Carême avec un léger profit. Mais leurs auberges, où font étape des paysans ou des marchands venus de plus loin, servent aussi de lieux de vente clandestine d'une viande détournée de sa destination finale qu'est la boutique du boucher ou du poulailler de Carême. De tels procédés d'accaparement et de concurrence déloyale expliquent sans doute les rancœurs des fermiers à l'égard de ces intermédiaires obligés et souvent haïs que sont les hôteliers et leur acharnement à poursuivre les coupables.

L'étude des arguments auxquels ont recours les accusés lors de leur interrogatoire a donc révélé les différentes stratégies de légitimation de la fraude et la façon dont la norme est tour à tour contestée ou réutilisée. Mais les pratiques finissent aussi par être à l'origine de réécritures, formelles ou factuelles, du droit et de la loi. Le législateur est ainsi obligé de composer avec la coutume aussi bien que le juge avec un principe non écrit d'équité. Certains accusés opposent en effet lors de leur défense la coutume au texte de la loi. Un boucher de Vaise explique ainsi que «depuis quinze ans qu'il demeure en Vaise et depuis les six derniers qu'il tient boutique il en a toujours usé de mesme et a toujours eu de la viande morte dans sa boutique soit en caresme ou en autre temps ${ }^{93}$. Le recours à 1'usage est particulièrement récurrent du côté des poulaillers, qui ont l'habitude de se débarrasser de leurs dernières volailles auprès du public ou du fermier dans les deux premiers jours du Carême, soit le mercredi des Cendres et le jeudi qui suit. L'un d'eux, Pierre Tissedre, chez qui sont saisies des volailles, se défend ainsi en expliquant «qu'a l'entrée du caresme lorsque le fermier leur demande la volaille ou gibier qu'ils ont de reste du carnaval ils ne font pas difficulté de la luy remettre en leur payant ce qu'elle leur a

\footnotetext{
ADR, BP 3624 (2 avril 1708).

ADR, BP 3624 (27 mars 1711).

ADR, BP 3624 (13 mars 1700).

ADR, BP 3624 (22 mars 1711).

ADR, BP 3624 (28 février 1700).
} 
cousté, mais il ne sont pas obligés de la porter chez ledit fermier a moins qu'il ne le demande, et c'est ce que ledit Piraud [fermier] n'a point fait a luy qui respond, ainsy il a esté obligé de garder celles qu'on luy a trouvées jusques a present, sachant bien qu'il ne luy est pas permis de la débiter a d'autres qu'audit fermier ${ }^{94}$. Lorsqu'un usage est profondément ancré dans le temps, il peut finir par s'imposer au législateur, parfois au détriment d'une loi plus récente, et de cette façon être normalisé, institutionnalisé ${ }^{95}$. C'est ce qui se produit en 1677 , lorsque les poulaillers obtiennent la suppression de la règle fraîchement établie qui leur interdit de vendre leurs dernières volailles pendant les deux jours qui suivent le mardi gras. L'argument qu'ils utilisent et qui se révèle décisif est que cette faculté «a toujours esté ainsy pratiquée et se pratique mesme a l'esgard du boucher ${ }^{96}$.

Par ailleurs, l'étude du montant des amendes infligées au moment du verdict révèle de telles disproportions avec celui des peines théoriquement encourues qu'elle oblige à réaffirmer une fois encore la forte distinction à opérer entre le texte de la loi et sa traduction dans la réalité. De 30 livres dans les années 1660, l'amende encourue - ou requise par le fermier - passe à 100 livres en 1688, puis à 150 livres en 1693 pour atteindre 250 livres en 1706. Une telle évolution incite à conclure de prime abord à un durcissement de la répression, éventuellement révélateur d'une amplification du phénomène du marché noir. Mais l'étude des condamnations effectives révèle en réalité l'extrême stabilité du montant des amendes infligées, qui reste globalement compris pour la période étudiée entre 5 et 30 livres. La faiblesse de celui-ci par rapport aux peines encourues ne renvoie-t-elle pas en outre à un principe informel d'équité, principe qui suspend ou infléchit la loi quand elle impose des conditions trop dures et qui semble parfaitement présent dans l'esprit de ceux qui se chargent de la faire appliquer ?77 C'est aussi sur ce type de négociations entre le législateur et les acteurs sociaux que repose en définitive le maintien de l'ordre public ${ }^{98}$. On peut s'interroger dans ce contexte sur l'effet véritablement dissuasif des règlements, le profit tiré de la pratique du marché noir pendant les six semaines du Carême pouvant compenser aisément la perte encourue en cas d'amende. N'y a-t-il pas ici encore, et cette fois avec la complicité des autorités, affaiblissement et «réécriture» factuelle d'une norme inapplicable dans sa lettre?

Contournée, modifiée, affaiblie, la règle est aussi - et c'est le cas extrême - régulièrement bafouée par ceux-là mêmes qui sont censés la faire appliquer. Il n'est pas rare que les commis aux portes se livrent ainsi, par complicité avec des bouchers contrevenants, à des vexations à l'égard du fermier ${ }^{99}$, voire qu'ils prennent personnellement une part active au marché noir: un des commis aux portes de la CroixRousse rétorque ainsi au fermier «qu'estant commis il estoit privilégié pour entrer de la viande et qu'il ne luy raisonnat pas davantage ${ }^{100}$. L'implication dans ces pratiques illicites de ceux qui sont censés faire respecter la loi donne donc, là encore,

\footnotetext{
94 ADR, BP 3624 (18 mars 1688).

95 Raggio (1990), Revel (1995).

96 ADR, BP 3624 ( $1^{\text {et }}$ mars 1677$)$.

97 Ago (1999, p. 393). Sur la question des tensions entre droit et équité, je tiens à remercier Renata Ago pour ses précieuses suggestions faites lors de la présentation de cette recherche au colloque organisé par l'Internationaal Instituut voor Sociale Geschiedenis à Amsterdam en avril 2000.

98 Napoli (1998, p. 127).

99 ADR, BP 3624 (affaire Giraud-Conchon-Laplatinière, mars 1700).

100 ADR, BP 3624 (4 mars 1692).
} 
une idée des enjeux économiques qu'elles impliquent. De la même façon, l'attribution de la ferme se charge au cours du XVIII ${ }^{\text {eme }}$ siècle de tels enjeux financiers - elle atteint en 1768 un montant de 40000 livres - que c'est le processus d'adjudication lui-même qui finit par être complètement fausse ${ }^{101}$. Les mancuvres des bouchers, contraints de s'associer à plusieurs pour pouvoir l'emporter, dépassent alors le niveau des stratégies individuelles. Les enchères officielles sont trafiquées et cachent des collusions entre les participants, qui s'accordent par exemple pour ne pas dépasser une somme qu'ils ont fixée. Le droit de débiter la viande en Carême devient ainsi le monopole d'une poignée de familles fortement liées entre elles et, par un système de conventions, avec des bouchers des quatre boucheries de la ville, des marchands de bétail, des tanneurs, etc. Les interdits religieux ne servent finalement plus, dans ce contexte, que de prétextes à la constitution de réseaux suscités par des intérêts purement économiques.

L'incapacité des autorités à contrôler le phénomène du marché parallèle est finalement révélatrice d'une contradiction profonde entre les règlements - expression des interdits catholiques - et les usages consuméristes d'une partie de la population lyonnaise $e^{102}$. Le marché parallèle de la viande en Carême n'est en cela qu'une des multiples facettes d'une «économie de l'illicite» qui sous bien d'autres aspects innerve toute la société d'Ancien Régime. Inscrit dans des espaces spécifiques, le phénomène fait apparaitre des résistances complexes face à la loi, révélatrices d'intérêts économiques - qui vont de la stratégie de survie individuelle au brigandage à grande échelle - aussi bien que de motivations religieuses ou culturelles (diffusion de nouvelles pratiques alimentaires signalant un rapport plus libre à la religion). Il met aussi en lumière, à travers les processus d'échanges continuels et dynamiques entre les individus et la norme, l'existence autour de la loi de tout un savoir social qui fait que celle-ci est concrètement et en permanence contestée, contournée, réinterprétée et, en un sens, recréée par les pratiques.

Anne MONTENACH

Université de Provence (Aix-Marseille I) Secteur Lettres-Sciences Humaines / U.F.R.

Civilisations et humanités

Département d'histoire 29 , avenue Robert Schuman 13621 Aix-en-Provence Cedex montenac@club-internet.fr

w1 Je reprends ici l'étude de Maurice Garden (1967, p. 70).

102 Roche (1995, pp. 10-11). 


\section{BIBLIOGRAPHIE}

Abad, R., Un indice de déchristianisation? L'évolution de la consommation de viande à Paris en Carême sous l'Ancien Régime, Revue historique, 1999, CCCI, 2, pp. 237-275.

Ago, R., Una giustizia personalizzata. I tribunali civili di Roma nel XVII secolo, Quaderni Storici, 1999, XXXIV, 101, 2, pp. 389-412.

Beik, W., Urban Protest in Seventeenth-Century France: The Culture of Retribution, Cambridge, Cambridge University Press, 1997.

Cenutti, S., Normes et pratiques, ou de la légitimité de leur opposition, in Lepetit, B. (Dir.), Les formes de l'expérience. Une autre histoire sociale, Paris, Albin Michel, 1995, pp. 127-149.

Flandrin, J.-L., Montanari, M., Histoire de l'alimentation, Paris, Fayard, 1996.

Freundlich, F, Le jeu à Paris au XVIIreme siècle (1715-1800), Paris, Albin Michel, 1995.

Garcia, S., La fraude forcée, Actes de la Recherche en Sciences Sociales, 1997, 118, pp. 8191.

Garden, M., Bouchers et boucheries de Lyon au XVIII ${ }^{\text {ème }}$ siècle, $92^{e}$ Congrès national des sociétés savantes, Strasbourg, 1967, tome 2, pp. 47-80.

Garnot, B. (Dir.), La petite délinquance du Moyen-Âge à l'époque contemporaine, Dijon, Éditions universitaires de Dijon, 1998.

Kuhen, T., Law, Family and Women. Toward a Legal Anthropology of Renaissance Italy, Chicago, 1991.

Levi, G., L'eredità immateriale : carriera di un esorcista nel Piemonte del seicento, Torino, Einaudi, 1985.

Lombardini, S., Raggio, O., Torre, A. (Dir.), Conflitti locali e idiomi politici, Quaderni Storici, 1986, XXI, 63, p. 3.

Martin, O., La conversion protestante à Lyon (1659-1687), Paris, Droz, 1986.

Napoli, P., Police et société. La médiation symbolique du droit, Enquête, 1998, 7, pp. 127144.

Péronnet, M., La broche, l'épée et le goupillon: des interdits alimentaires ordonnés par Dieu, l'Église et le Roi, in Blanchard, A., Michel, H., Pélaquier, E. (Dir.), De l'herbe à la table. La viande dans la France méridionale à l'époque moderne, Montpellier, Université Paul Valéry-Montpellier III, 1994, pp. 251-283.

Raggio, O., Faide e parentele. Lo stato genovese visto dalla Fontanabuona, Torino, Einaudi, 1990.

Revel, J., L'institution et le social, in Lepetit, B. (Dir.), Les formes de l'expérience. Une autre histoire sociale, Paris, Albin Michel, 1995, pp. 63-84.

Roche, D., Préface, in Freundlich, F., Le jeu à Paris au XVIII ${ }^{\text {eme }}$ siècle (1715-1800), Paris, Albin Michel, 1995.

Schlumbohm, J., Gesetze, die nicht durchgesetzt werden - ein Strukturmerkmal des frühneuzeitlichen Staates?, Geschichte und Gesellschaft, 1997, 23, 4, pp. 647-663.

Thompson, E.P., The moral economy of the english crowd in the eighteenth century, Past and Present, 1971, 50, pp. 76-136 (L'économie morale de la foule dans l'Angleterre du XVIII'me siècle, in Gauthier, F., Ikni, G.-R., La guerre du blé au XVIII ${ }^{\text {me }}$ siècle, Montreuil, Les Éditions de la Passion, 1988).

Vigarello, G., Le sain et le malsain, Paris, Seuil, 1993. 